\title{
Academic career after treatment for acute lymphoblastic leukaemia
}

\author{
A Kingma, L A J Rammeloo, A van der Does-van den Berg, L Rekers-Mombarg, A Postma
}

\begin{abstract}
Aim-To evaluate academic career in long term survivors of childhood acute lymphoblastic leukaemia (ALL), in comparison to their healthy siblings.

Patients-Ninety four children treated for ALL with cranial irradiation 18 or $25 \mathrm{~Gy}$ and intrathecal methotrexate as CNS prophylaxis. Median age at evaluation was 20 years; median follow up since diagnosis was 15 years at the time of the study.

Methods-Patients and their 134 siblings completed a questionnaire on school career. The percentage of referrals to special primary schools for learning disabled, and the final level of secondary education in patients and siblings were compared, using a six point classification. Within the patient group, the effect of possible risk factors (age at diagnosis, irradiation dose, and gender) was investigated.
\end{abstract}

Results-Significantly more patients than siblings were placed in special educational programmes. A significant difference was found for level of secondary education. No effect of gender or irradiation dose was found, but younger age at diagnosis was significantly related to both referrals and school levels.

Children's Cancer

Center, Department of Pediatrics, University Hospital of Groningen, PO Box 30.001, 9700

RB Groningen,

Netherlands

A Kingma

A Postma

Department of Pediatrics and Department of Clinical

Epidemiology and Biostatistics, Free University Hospital of Amsterdam, De Boelelaan 1117, 1081 HV Amsterdam,

Netherlands

L A J Rammeloo

L Rekers-Mombarg

Dutch Childhood Leukemia Study Group, PO Box 43515, 2504 AM The Hague,

Netherlands

A van der Does-van den Berg

Correspondence to:

Dr Kingma

email:

W.A.Kamps@medrug.nl

Accepted 4 February 2000 survival of children with ALL. But growing evidence emerged that giving such treatment could result in neuropsychological impairment, and cranial irradiation has now been eliminated from most treatment protocols for standard risk ALL. ${ }^{12}$

Cognitive deficits in irradiated children with ALL have been found in various domains, including intelligence, learning and memory, attention, cognitive speed, academic achievement, and executive and visual-motor functioning. ${ }^{3-8}$ The underlying cerebral pathology of such impairment probably includes necrotising leucoencephalopathy and mineralising microangiopathy. ${ }^{9}$ Neuroradiological studies in ALL survivors who received cranial irradiation have shown white matter abnormalities, calcifications, and parenchymal atrophy. ${ }^{10}{ }^{11}$ Longitudinal neuropsychological research in children treated for ALL at a young age (under the age of 7 years) have been performed in our institution since the early 1980s. Children who received cranial irradiation and intrathecal methotrexate (MTX) performed significantly worse than normal controls on a variety of measures, both two and six years after CNS prophylaxis. Magnetic resonance imaging (MRI) of the brain revealed abnormalities in $63 \%$ of cases. ${ }^{12}$ With a mean follow up of 13 years, school career was evaluated in 28 patients and their 48 normal siblings. Leukaemia survivors reached significantly lower levels of secondary education than their siblings and were more often referred to special schools for learning disabled. However, only young patients were eligible for this evaluation, and young age is considered an additional risk factor for cognitive impairment when receiving cranial irradiation. ${ }^{13}$

Another limitation was that the influence of risk factors such as cranial irradiation dose, sex, and age at diagnosis could not be determined because of small patient numbers. To date, results are not unequivocal with regard to the effects of risk factors on cognitive functioning, despite abundant neuropsychological research in ALL patients. ${ }^{14}$ Limitations of many studies include absence of normal controls and follow up not long enough for evaluation of final school performance. To avoid our former selection bias of young age and to obtain larger patient numbers for multivariate analysis, a nationwide study was designed. We wished to compare school career in ALL patients who received cranial irradiation and their normal siblings, so that patients could be compared to youngsters from the same socioeconomic and intellectual background. We expected patients' school achievement to be inferior to the accomplishment of their siblings; additionally, hypotheses concerning the effect of various risk factors were tested.
Children were eligible for this study if they fulfilled the following criteria: (1) in continuous

complete remission after treatment according

\section{Methods}

This study was clustered with a nationwide cardiac evaluation among long term ALL survivors diagnosed from 1979 to 1984 and treated with cranial irradiation. ${ }^{15}$ Patients were invited to participate by mail. Written informed consent was obtained according to guidelines of the 11 participating institutions.

\section{SUBJECTS}


Table 1 Characteristics of patients treated for ALL with cranial irradiation and chemotherapy, and their siblings

\begin{tabular}{llll}
\hline & All patients $(n=94)$ & Siblings $(n=134)$ & $\begin{array}{l}\text { Patients with siblings } \\
(n=85)\end{array}$ \\
\hline $\begin{array}{l}\text { Male/female } \\
\begin{array}{c}\text { Median age (y; mth) at } \\
\text { diagnosis (range) }\end{array}\end{array}$ & $40 / 54$ & $65 / 69$ & $36 / 49$ \\
$\begin{array}{c}\text { Patients with 25/18 Gy } \\
\text { irradiation }\end{array}$ & $72 / 22$ & n.a. & $4 ; 5(1 ; 2-14 ; 9)$ \\
$\begin{array}{c}\text { Median age (y; mth) at } \\
\text { evaluation (range) }\end{array}$ & $20 ; 1(14 ; 8-31 ; 6)$ & n.a. & $65 / 20$ \\
\hline n.a., not applicable. & $19 ; 0(14 ; 0-32.0)$ & $19 ; 7(14 ; 4-31 ; 2)$ \\
\hline
\end{tabular}

to protocol ALL 5 for non-high risk patients or the preceding pilot study from the Dutch Childhood Leukemia Study Group (DCLSG); (2) no congenital heart disease or Down's syndrome; (3) treated in a university related paediatric oncology centre. Ninety four of 146 eligible patients (64\%) agreed to participate in the school evaluation. CNS prophylaxis consisted of cranial irradiation and intrathecal MTX and prednisolone (five doses: 12.5 $\mathrm{mg} / \mathrm{m}^{2}$ with a maximum of $15 \mathrm{mg}$ MTX and the same dose of prednisolone per dose). Total cranial irradiation dose was $25 \mathrm{~Gy}$ in children aged at least 2 years; in children under 2 years, 20 Gy was given from 1979 to 1983 and 18 Gy from 1983 to 1984 . Daily dose fraction in all children was $1.5 \mathrm{~Gy}$. Treatment continued with maintenance and consolidation therapy for a total duration of 24 months. ${ }^{15}$

EVALUATION AND STATISTICAL ANALYSIS

Patients and their siblings (or parents) completed a questionnaire which contained five items on school career developed for the neuropsychological research in our institution. Firstly, it was registered whether children attended regular primary schools or special schools for learning disabled. Secondly, the level of secondary education was classified using a six point scale. Category 1 is the lowest, category 6 the highest, and category 4 is the mean level of secondary education in the Netherlands. ${ }^{16}$ Special education for learning disabled comprises categories 1 and 2, representing schools for children with defective and low normal intelligence respectively. Table 2 presents equivalents of the six categories for the UK educational system. Children still at secondary school received the same classification as children who had passed the final examination.

The following hypotheses were formulated and investigated using the indicated statistical tests.

(1) The percentage of referrals to primary schools for learning disabled is higher in patients than in siblings (Fisher exact test, one tailed)

(2) Mean level of secondary education in (subgroups of) patients is lower than in (subgroups of) siblings (Wilcoxon signed rank test, one tailed). If a patient had more than one sibling, he or she was compared to the mean level of the siblings

(3) To examine the effects of three risk factors (age at diagnosis, cranial irradiation dose, and gender) on referral to special schools and on level of secondary education within the patient group, univariate and multivariate linear regression analyses were performed, followed by three way analysis of variance (ANOVA). We expected that lower age at diagnosis and higher cranial irradiation dose were related to higher referrals and lower educational levels, but no gender effect was anticipated.

Significance levels were established at $\mathrm{p} \leqslant$ 0.05. All statistical analyses were carried out with the Statistical Package for Social Sciences. ${ }^{17}$

\section{Results}

Firstly, demographic variables were compared. The enrolled 95 participants did not significantly differ from the 51 eligible nonparticipants with regard to age at diagnosis, cranial irradiation dose, and gender. Eighty five of 94 patients had one or more siblings. There were also no demographic differences between patients and siblings and between patients with and without siblings. Table 1 shows characteristics of patients. Mean follow up since diagnosis was 15 years at the time of the study.

\section{REFERRALS TO SPECIAL PRIMARY SCHOOLS FOR} LEARNING DISABLED

All children had finished primary school. Twelve patients and one sibling were placed in special educational classes. Statistically, this difference is highly significant $(p<0.0001)$, confirming hypothesis 1 . However, considering this low number of referrals, the validity of this result can be questionned.

MEAN LEVEL OF SECONDARY EDUCATION

Twenty patients were still at secondary school; 74 had passed the final examination. Concurrent with hypothesis 2, a significant $(p<0.0001)$ difference in educational level was found when comparing patients and siblings (table 2). The overall mean level for patients was 3.4 (range 1-6) and the overall mean level for siblings 4.5 (range 3-6). Table 2 also gives the results for different subgroups of patients and their respective siblings. Significant differences were seen for all patientsibling comparisons except for 21 patients aged over 7 years at the time of diagnosis, when mean level of education no longer differed from that of their siblings.

\section{EVALUATION OF THREE RISK FACTORS WITHIN} THE PATIENT GROUP

Using univariate linear regression analysis, no significant effect of gender or cranial irradiation dose was found, either on referral to special schools or on level of secondary education. As expected, younger age at diagnosis was significantly related to both referrals $(p=0.04)$ and educational level $(p=0.003)$. For the latter, age at diagnosis explained $9 \%$ of variance.

Multivariate regression analysis with age at diagnosis, sex, and cranial irradiation dose as independent variables was only applicable for 
Table 2 Level of secondary education * in patients treated for ALL with cranial irradiation and chemotherapy; comparisons of (subgroups of) patients to (subgroups of) their siblings

\begin{tabular}{|c|c|c|c|c|}
\hline & & $\begin{array}{l}\text { Mean level } \\
\text { (SD) }\end{array}$ & Range & $p$ value \\
\hline All patients & & $3.4(1.1)$ & $1-6$ & \\
\hline Their siblings & $\mathrm{n}=85$ pairs & $4.5(1.1)$ & $3-6$ & $\mathrm{p}<0.0001$ \\
\hline Male patients & & $3.4(1.4)$ & $1-6$ & \\
\hline Their male siblings & $\mathrm{n}=20$ pairs & $4.5(1.1)$ & $2-6$ & $\mathrm{p}=0.0021$ \\
\hline Female patients & & $3.6(1.1)$ & $1-6$ & \\
\hline Their female siblings & $\mathrm{n}=31$ pairs & $4.5(1.0)$ & $3-6$ & $p=0.0006$ \\
\hline Patients who received 25 Gy & & $3.4(1.2)$ & $1-6$ & \\
\hline Their siblings & $\mathrm{n}=65$ pairs & $4.5(1.1)$ & $3-6$ & $p=0.0001$ \\
\hline Patients who received $18 \mathrm{~Gy}$ & & $3.5(0.9)$ & $2-6$ & \\
\hline Their siblings & $\mathrm{n}=20$ pairs & $4.3(1.1)$ & $3-6$ & $\mathrm{p}=0.0034$ \\
\hline Patients aged $0-3$ y at diagnosis & & $2.9(1.0)$ & $1-5$ & \\
\hline Their siblings & $\mathrm{n}=38$ pairs & $4.4(1.0)$ & $3-6$ & $\mathrm{p}<0.0001$ \\
\hline Patients aged $4-6$ y at diagnosis & & $3.7(1.0)$ & $2-6$ & \\
\hline Their siblings & $\mathrm{n}=26$ pairs & $4.7(1.1)$ & $3-6$ & $\mathrm{p}=0.0003$ \\
\hline Patients aged $\geqslant 7 \mathrm{y}$ at diagnosis & & $4.0(1.0)$ & $2-6$ & \\
\hline Their siblings & $\mathrm{n}=21$ pairs & $4.4(1.2)$ & $3-6$ & $\mathrm{p}=0.1823$ \\
\hline
\end{tabular}

${ }^{\star}$ On a six point scale: $1=$ lowest; $4=$ Dutch population mean; $6=$ highest:

(1) Exclusive special education for moderate/severe learning disabilities (mental disabled with low IQ) (in Dutch: MLK or ZMLK and VSO(Z)MLK)

(2) Exclusive special education for mild learning disabilities or behavioural disorders with (sub)normal IQ (in Dutch: LOM, VSO-LOM and IVBO)

(3) Regular secondary school education for lower IQ levels (in Dutch: LBO or VBO)

(4) Regular secondary school education for average IQ levels (= Dutch population mean) (in Dutch: MAVO)

(5) Regular secondary school education for higher IQ levels (in Dutch: HAVO)

(6) Regular secondary school education, higher level preparing for university (A levels, college school) (in Dutch: VWO).

evaluation of secondary education. Again, a significant $(p=0.003)$ effect was seen for age at diagnosis only. A three way ANOVA examining main and interaction effects of risk factors on school level again showed a significant $(p=0.013)$ main effect for age. Thus, hypothesis 3 was partly confirmed.

The regression analysis was repeated with exclusion of patients placed in school categories 1 and 2 (without children referred to schools for learning disabled), to assess whether or not the age effect was only determined by patients with obvious defective achievement. However, younger age at diagnosis was still the only factor significantly $(p=0.047)$ related to lower school level. Hence, young age contributed to lower school levels in both patients with normal (categories 3-6) or impaired (categories 1 and 2) academic functioning, although the significance of the age effect decreased after exclusion of the latter.

\section{Discussion}

The results of this nationwide study confirm an earlier institutional report that ALL treatment with cranial irradiation as CNS prophylaxis is associated with significantly poorer school performance in patients compared to siblings. This is shown by both a higher referral to special primary schools for learning disabled and a lower level of accomplished secondary education in ALL survivors, confirming our hypotheses. Previous studies have shown controversial results considering the influence of risk factors on cognitive functioning. This is probably because of differences and restrictions in study design and methodology. In the present study, young age at diagnosis was a distinct and single factor determining school career many years after treatment. Young children are considered as particularly vulnerable with regard to injurious treatment, although the definition of "young" varies from 2 to 7 years. Myelinisa- tion, synaptogenesis, and dendrite branching may all be affected by cranial irradiation and intrathecal MTX. $^{18}{ }^{19}$ Radiotherapy and chemotherapy can cause diffuse white matter injury which has been associated with the nonverbal learning syndrome. ${ }^{20}$ Such learning disability could be one of the reasons for the poorer school career among patients in this study. In agreement with Mulhern et al, cranial irradiation dose, neither alone nor in combination with young age, was associated with worse academic achievement. ${ }^{21}$

Alhough several investigators have studied gender effects, clear consensus has yet to emerge.$^{13}$ If sex is considered a risk factor, cognitive deficits have been more frequently found in female than male patients. ${ }^{22}{ }^{23}$ This can be explained by differences in brain lateralisation (females tend to show less hemispheric lateralisation in function), myelination rate (higher in girls in early years), and ratio of grey to white matter (higher in females). ${ }^{24-26}$ In contrast to the Waber studies, however, no gender effect was found in the present study. ${ }^{22}$

Our questionnaire appears to be an ecologically valid method for global investigation of the long term effects of treatment. The usefulness of such an evaluation is also in line with results of studies evaluating academic sequelae of other kinds of acquired brain damage sustained during childhood. ${ }^{27}$

Two aspects of the study methodology deserve comment. Firstly, 36\% of the patients did not reply to the mailed questionnaire. We are not sure whether or not the nonparticipants have caused bias in our results. Considering the even distribution of distinctive factors (age, cranial irradiation dose, and gender) among the non-responders and participants, bias in results is not very likely. If bias occurred, it is most probable that the nonresponders had even worse educational outcome than the participants, augmenting the differences between patients and siblings. Secondly, it cannot be ruled out that school career in the sibling controls was also affected by the cancer experience in the families. However, this is not plausible given the mean level of education in the siblings group which equalled the Dutch population mean. Apart from emotional influences, use of siblings controls has the advantage of equal socioeconomic background and comparable cognitive potential, which are important determinants of academic career. Unfortunately, no information was available for school career in siblings and patients prior to diagnosis.

School career is not solely determined by cognitive capacities, but can also be influenced by emotional status. Children treated for ALL could be at risk of developing psychological problems resulting in cognitive impairment. ${ }^{28}$ We do not assume, however, that emotional functioning was a significant factor in the present results because of the following: (1) patients older than 7 years at diagnosis had the same accomplished level of secondary education as their siblings; and (2) we have no clinical indication that the psychological impact of disease and treatment is stronger in younger 
than older patients. Furthermore, in a pilot study in long term ALL survivors treated with chemotherapy only at a young age in our institution, no differences in school career were seen in comparison to their siblings. ${ }^{12}$ Thus, cranial irradiation below the age of 7 , which includes $70 \%$ of all ALL patients, appears to be the decisive factor for poorer academic career. Finally, school absenteeism could interfere with optimal academic career, but given 13 years follow up it is not likely that absenteeism significantly contributed to these results.

The majority of patients in this study was not mentally retarded and it may be questioned whether the 1.1 point difference in school level in comparison to siblings is clinically important. Many patients perceive their mild scholastic arrears as a major late effect of leukaemia treatment, and this is particularly true if confronted with smarter siblings. To illustrate the meaning of a one point difference we offer two examples from the Dutch school practice: (1) the difference between school categories 2 and 3 signifies a distinction between special and regular education with accordingly less or more vocational opportunities; (2) the difference between categories 6 and 5 determines whether or not you can go to university, which may result in a sense of inferiority for the ALL survivor with level 5 if he or she is a member of a university educated family.

Cranial irradiation has now been eliminated from most treatment protocols, but patients with meningeal leukaemia or receiving bone marrow transplantation may still be confronted with limitations in school career as described above. Such children require special educational assistance and teachers and parents should be alerted to the possibility of a break in school career. The neuropsychological late effects of new combinations of chemotherapy remain to be seen. There is growing concern about the adverse sequelae on cognitive development of some drugs used in leukaemia treatment, such as MTX, dexamethasone, and cytosine arabinoside. ${ }^{29} 30$ The present evaluation should therefore be repeated among children treated on subsequent DCLSG ALL protocols with chemotherapy alone.

Ideally, repeated and exhaustive neuropsychological assessments would yield the most useful data to evaluate the cognitive consequences of current and new treatment protocols. If such examinations are not feasible, our questionnaire may be a good alternative. In any country with an educational system which unequivocally and unalteredly marks academic performance throughout the country for years, registration of referral to special classes and final level of secondary education may improve follow up in long term survivors.

We gratefully acknowledge David Gooding from North Stifford, UK for his considerable efforts in comparing Dutch school levels with the UK educational system.

1 Pinkel D. Lessons from 20 years of curative therapy of childhood acute leukemia. Br f Cancer 1992;65:148-53.

2 Veerman AJP, Hählen K, Kamps WA, et al. High cure rate with moderately intensive treatment regimen in non-high with moderately intensive treatment regimen in non-high protocol ALL VI (1984-1988) from the Dutch Childhood Leukemia Study Group. F Clin Oncol 1996;3:911-18.
3 Williams J, Davis KS. Central nervous system prophylactic reatment for childhood leukemia: neuropsychological outcome studies. Cancer Treat Rev 1986;13:113-27.

4 Fletcher JM, Copeland DR. Neurobehavioral effects of central nervous system prophylactic treatment of cancer in children. F Clin Exp Neuropsychol 1988;10:495-537.

5 Cousens P, Waters B, Said J, Stevens M. Cognitive effects of cranial irradiation in leukemia: a survey and meta-analysis. f Child Psychol Psychiatry 1988;6:839-52.

6 Stebhens JA, Kaleita TA, Noll RB. CNS prophylaxis of childhood leukemia: what are the long term neuropsychoogical, neurological and behavioral effects? Neuropsychol Rev 1991;2:147-77.

7 Madan-Swain A, Brown RT. Cognitive and psychosocial sequelae for children with acute lymphoblastic leukemia and their families. Clin Psychol Rev 1991;11:267-94.

8 Roman DD, Sperduto PW. Neuropsychological effects of cranial radiation: current knowledge and future directions. Int F Radiat Oncol Biol Phys 1995;31:983-98.

9 Bleyer WA, Griffin WG. White matter necrosis, mineralizing microangiopathy and intellectual abilities in survivors of childhood leukemia: associations with CNS irradiation and methotrexate therapy. In: Gilbert HA, Kagan AR, eds. Radiation damage to the nervous system. New York: Raven Press, 1980:155-74.

10 Kingma A, Mooyaart EL, Kamps WA, Nieuwenhuizen P, Wilmink JT. Magnetic resonance imaging of the brain and neuropsychological evaluation in children treated for acute ymphoblastic leukemia at a young age. Am f Pediatr Hematol Oncol 1993;15:231-8.

11 Packer RJ, Meadows AT, Rorke LB, Goldwein JW, D’Angio G. Long-term sequelae of cancer treatment on the central
nervous system. Med Pediatr Oncol 1987;15:241-53.

12 Kingma A, Van Dommelen RI, Mooyaart EL, Wilmink JT, Deelman BG, Kamps WA. Neuropsychological assessment, academic career and magnetic resonance imaging of ment, academic career and magnetic resonance imaging of mia with or without cranial irradiation (submitted)

13 Eiser C. Cognitive deficits in children treated for leukemia. Arch Dis Child 1991;66:164-8.

14 Butler RW, Copeland DR. Neuropsychological effects of central nervous system prophylactic treatment in childhood leukemia: methodological considerations. I Pediatr Psychol 1993;18:319-38.

15 Van der Does-van den Berg A, Van Wering ER, Suciu S, et al. Effectiveness of rubidomycin in induction therapy with vincristine, prednisone and L-asparaginase for standard risk childhood acute lymphocytic leukemia: results of a Dutch phase III study (ALL V). Am 7 Pediatr Hematol Oncol 1989;11:125-33.

16 CBS. Enquete beroepsbevolking 1995 Centraal Bureau voor de Statistiek. Heerlen: CBS, Div. Sociaal-Economische Statistieken, 1996.

17 Norusis MJ. SPSS for Windows. Base system user's guide. Release 6.0. Chicago: SPSS Inc., 1993.

18 Dietrich RB, Bradley WG, Zaragoza EJ, Otto RJ, Taira RK, Wilson GH, Kangarloo H. MR evaluation of early myelination patterns in normal and developmentally delayed infants. AFNR 1988;9:69-76.

19 Aram DM, Eisele JA. Plasticity and recovery of higher cognitive functions following early brain injury. In: Rapin J, Segalowitz SJ, eds. Handbook of neuropsychology. Amsterdam: Elsevier, 1992

20 Rourke BP. Syndrome of nonverbal learning disabilities: the final common pathway of white matter disease/ dysfunction? Clin Neuropsychol 1987;1:209-34.

21 Mulhern RK, Fairclough D, Ochs J. A prospective comparison of neuropsychologic performance of children surviving leukemia who received 18-Gy, 24 Gy, or no cranial irradialeukemia who received 18-Gy, 24 G

22 Waber DP, Tarbell NJ, Kahn CM, Gelber RD, Sallan SE. The relationship of sex and treatment modality to neuropsychologic outcome in childhood acute lymphoblastic leukemia. f Clin Oncol 1992;10:810-17.

23 Bleyer WA, Fallavollita J, Robinson LL. Influence of age, sex and concurrent intrathecal methotrexate therapy on intellectual function after cranial irradiation during childhood. A report from the Children's Cancer Study Group. Int $\mathcal{F}$ Pediatr Hematol Oncol 1990;7:29-38.

24 McGlone J. Sex differences in human brain asymmetry: a critical survey. Behav Brain Sci 1980;3:215-63.

25 Gur RC, Gur RE, Obrist WD, Hungerbuhler IP, Younkin D, Rosen A, Skolnick B, Reirich M. Sex and handedness differences in cerebral blood flow during rest and cognitive activity. Science 1982;217:659-60.

26 Witebon SF. Neural sexual mosaicism: sexual differentiation of the human temporo-parietal regions for functional of the human temporo-parietal regions for functiona

27 Ewing-Cobbs L, Fletcher JM, Levin HS, Iovino I, Miner ME. Academic achievement and academic placement following traumatic brain injury in children and adolescents: a two-year longitudinal study. $\mathcal{F} \mathrm{Clin} \operatorname{Exp} \mathrm{Neu}$ ropsychol 1998;20:769-81.

28 Van Dongen-Melman JEWM. On surviving childhood cancer: late psychosocial consequences for patients, parents, and siblings. Thesis. Rotterdam: Erasmus University, 1995.

29 Ochs J, Mulhern RK, Fairclough D, et al. Comparison of neuropsychologic functioning and clinical indicators of neurotoxicity in long term survivors of childhood leukemia given cranial irradiation or parenteral methotrexate: a progiven cranial irradiation or parenteral metho
spective study. F Clin Oncol 1991;1:145-51.

30 Brown RT, Madan-Swain A, Pais R. Chemotherapy for Brown RT, Madan-Swain A, Pais R. Chemotherapy for
ALL: congnitive and academic sequelae. $\mathcal{F}$ Pediatr 1992; 121:885-9. 